\title{
Comparative study of the efficacy and safety of minimally invasive interlaminar full-endoscopic discectomy versus conventional microscopic discectomy in single-level lumbar herniated intervertebral disc (ENDO-F Trial): A multicenter, prospective, randomized controlled trial protocol
}

Jin-Sung Kim

The Catholic University of Korea

Jun Ho Lee

Kyung Hee University Medical Center

Junseok Bae

Chungdam Wooridul Spine Hospital

Dong Chan Lee

Wiltse Memorial Hospital

Sang-Ha Shin

Chungdam Wooridul Spine Hospital

Han Joong Keum

Chungdam Wooridul Spine Hospital

Young Soo Choi

Chungdam Wooridul Spine Hospital

Sang Soo Eun

Chungdam Wooridul Spine Hospital

Seung Ho Shin

Wiltse Memorial Hospital

Hyun Jin Hong

Wiltse Memorial Hospital

Ji Yeon Kim

Wiltse Memorial Hospital

Tae Hyun Kim

Wiltse Memorial Hospital

Woojung Lim

The Catholic University of Korea

Junghoon Kim 
The Catholic University of Korea

\section{Sang-Min Park}

Seoul National University College of Medicine and Seoul National University Bundang Hospital

\section{Hyun-Jin Park}

Hallym University College of Medicine

Hong-Jae Lee ( $\nabla$ kosailee73@gmail.com )

The Catholic University of Korea

\section{Study protocol}

Keywords: conventional microscopic discectomy, interlaminar full-endoscopic discectomy, lumbar disc herniation, study protocol, minimally invasive surgery

Posted Date: September 16th, 2021

DOl: https://doi.org/10.21203/rs.3.rs-877467/v1

License: (c) (i) This work is licensed under a Creative Commons Attribution 4.0 International License. Read Full License

Version of Record: A version of this preprint was published at Journal of Orthopaedic Surgery and Research on March 28th, 2022. See the published version at https://doi.org/10.1186/s13018-022-030521. 


\section{Abstract}

\section{Background}

Advances in minimally invasive surgery have expanded the indications for interlaminar full-endoscopic discectomy. Although the clinical outcomes for this approach may be equivalent to those of conventional microscopic discectomy, the supporting evidence is based on small, single-center, prospective, and retrospective studies. Therefore, a multicenter, randomized controlled trial is warranted.

\section{Methods}

This will be a prospective, multicenter, randomized controlled trial comparing the efficacy and safety of interlaminar full-endoscopic discectomy to those of conventional microscopic discectomy. The trial will enroll 100 participants with a lumbar disc herniation, 50 in each group. The primary outcome will be the Oswestry Disability Index (ODI) score at 12 months post-surgery. Secondary outcomes will be: back and leg pain (visual analog scale); the ODI; the EuroQol-5-Dimension score; patient satisfaction; and walking distance/time and time to return to daily activities post-surgery. Surgical outcomes will include postoperative drainage, operative time, duration of hospital stay, postoperative creatine kinase level as an indicator of muscle injury, and postoperative scarring. Postoperative magnetic resonance imaging, computed tomography, and simple radiography will be performed to evaluate radiographic outcomes between the two surgical approaches. Surgery-related complications and adverse effects will be evaluated as safety outcomes. A single assessor at each participating hospital, blinded to group allocation, will assess the enrolled participants at baseline, 2 weeks and 3, 6, and 12 months postoperatively.

\section{Discussion}

This trial is designed to determine whether interlaminar full-endoscopic discectomy is clinically comparable to microscopic discectomy to treat lumbar disc herniations. All efforts will be made to reduce bias, including adequate sample sizes, blinded analyses, and multicenter prospective registration. The outcomes will inform practice, providing the evidence needed for the use of interlaminar full-endoscopic over microscopic discectomy by confirming the potential of this technique to improve patient satisfaction and clinical outcomes.

Trial registration

Clinical Research Information Service; cris.nih.go.kr. (KCT0006277); protocol version (v1, June 8, 2021)

\section{Background}

Discectomy is the most common surgical method for resolving lumbar radiculopathy caused by disc herniation and nerve root compression [1, 2]. Currently, microscopic discectomy is performed as a minimally invasive surgery, reducing the invasiveness of conventional open discectomy [3-5]. Minimally 
invasive spinal surgery has been developed with the use of a tubular retractor, microscope, and endoscope to achieve effective neural decompression while preserving the stabilizing structures of the spine [4-7]. Although technically more demanding, interlaminar full-endoscopic discectomy has significantly reduced surgical invasiveness, thereby expanding the indications for endoscopic surgery [5, 8-10]. Specifically, interlaminar full-endoscopic discectomy offers several advantages over conventional microscopic discectomy, including a smaller skin incision, and thus, less scarring as well as less muscle damage, a lower infection rate and volume of blood loss, a less painful recovery, and a shorter hospital stay $[3,11-17]$. Previous studies have reported that there are no differences in clinical outcomes between interlaminar endoscopic and microscopic discectomy [18-22]. However, the evidence regarding the advantages of interlaminar full-endoscopic discectomy is limited by the small number of patients included in these studies and the type of research design, namely retrospective, single-center prospective designs [7, 18-25]. Therefore, a multicenter, randomized controlled trial (RCT) is warranted.

To address this gap in evidence, we propose a multicenter, prospective RCT to compare the outcomes of interlaminar full-endoscopic versus microscopic discectomy. Our guiding hypothesis is that the efficacy and safety of interlaminar full-endoscopic discectomy and microscopic discectomy of the lumbar spine will be similar.

\section{Methods/design}

\section{Trial design}

This study aims to compare the outcomes of interlaminar full-endoscopic versus microscopic discectomy in a multicenter, assessor-blind, prospective RCT. Our methods have been approved by the institutional review boards of the participating hospitals (The Catholic University of Korea Seoul St Mary's Hospital; Kyung Hee University Medical Center; Chungdam Wooridul Spine Hospital; Wiltse Memorial Hospital; Seoul National University Bundang Hospital; Kangnam Sacred Heart Hospital; The Catholic University of Korea Daejeon St. Mary's Hospital).

\section{Participant population}

The study sample will be 100 adults, $20-80$ years of age, who present with radiating pain in the lower extremities due to a lumbar disc herniation. The sample size calculation is provided below. Fifty participants will be allocated to each group: the interlaminar full-endoscopic group and microscopic discectomy groups. The equivalence between the two groups at baseline will be ascertained. Participants will be recruited from the five participating hospitals.

\section{Inclusion criteria}

The inclusion criteria are as follows: age, 20-80 years; diagnosis of single-level lumbar disc herniation; radiating pain to the lower extremities, with a pain score of $>4$ on a 10-point visual analog scale (VAS); ability to follow instructions and to provide consent for participation; and willingness to comply with the trial's follow-up protocol. 


\section{Exclusion criteria}

The exclusion criteria are as follows: the presence of a spondylolisthesis (Meyerding grade $\geq \mathrm{II}$ ); history of lumbar spinal surgery at the same level; the presence of degenerative lumbar scoliosis (Cobb angle > $20^{\circ}$ ), other spinal diseases (e.g., ankylosing spondylitis, spine tumor, fracture, or neurologic disorders), and psychological disorders (e.g., dementia, intellectual disability, or drug abuse); and "any other" patient characteristic or disorders that the surgeons consider inappropriate for participation.

\section{Recruitment}

This is a multicenter prospective RCT, and each of the five participating hospitals will recruit trial participants from patients who decide to proceed with a one-level discectomy for lumbar disc herniation from June 2021 to December 2024; there will be no recruitment via the social media. The researchers from each of the five hospitals will screen potential participants to determine their eligibility. After providing informed consent, enrolled participants will undergo baseline assessments. The assessor will be blinded to the participants' personal information.

\section{Randomization and follow-up}

After completing the baseline assessments, participants will be block-randomized into either the control (microscopy) or intervention (endoscopy) group, using a 1:1 allocation ratio, with a block size of four. The randomization list will be computer-generated and integrated into a web-based electronic case report form (eCRF) platform (iCReaT; internet-based clinical research and trial, icreat.nih.go.kr) accessible only to the trial's authorized researchers. The randomization will be implemented independently at each participating hospital. Group allocation will be presented to the surgeons using consecutively numbered opaque envelopes. To evaluate the primary and secondary outcomes, follow-up assessments will be planned for each participant at 2 weeks and 3, 6, and 12 months after surgery. An independent researcher will perform the assessments at each time-point of follow-up, with phone interviews used under unavoidable circumstances in which in-person follow-up is not possible (Fig. 1).

\section{Blinding}

All the primary and secondary outcomes will be assessed at each participating hospital by a single assessor, who will be blinded to the group allocation. The surgeons and participants will know which procedure was performed (interlaminar full-endoscopic discectomy or microscopic discectomy); this information will not be revealed by either participants or surgeons to the assessor. If unblinding is required, based on the assessment findings, the assessor will be required to submit a justification to the trial team.

\section{Surgical interventions}

\section{Active intervention: Interlaminar full-endoscopic discectomy}


The technical procedure for interlaminar full-endoscopic discectomy [26], including recent updates, is well described in the literature $[3,4,27-31]$. This surgical approach is familiar to spine surgeons, being similar to the conventional posterior approach with the exception of having to create a working channel for the endoscope and spinal instrument $[3,4,30]$. The procedure is performed under general or spinal anesthesia with the participant placed prone. The surgical table is bent approximately at the level of the lower lumbar spine, with appropriate flexion of the hip and knees; this position widens the interlaminar window $[3,4]$. A 5-7 mm skin incision is created at the working channel's entry point, approximately $1 \mathrm{~cm}$ from the midline at the level of the symptomatic herniated disc, visualized by intraoperative $\mathrm{C}$-arm anteroposterior fluoroscopy imaging, as previously described [28]. The working channel is inserted and positioned at the target point over the ligamentum flavum with sufficient subdermal fascia dissection. After optimal positioning of the working channel at the targeted location, the endoscope is inserted into the working channel under sufficient irrigation with saline solution. [3, 4, 28, 30, 31].

Surgery is performed by inserting the required spinal surgical instruments (bipolar radiofrequency cauterization devices, burrs, Kerrison punches, and pituitary rongeurs) through the working channel. The paravertebral muscle is coagulated to identify the border of the interlaminar window $[3,4,28]$. The ligamentum flavum is resected using a punch or split using a probe at the level of the tip of the descending facet $[3,11,28,29]$. The discectomy is performed in a fully endoscopic, minimally invasive manner (Fig. 2).

\section{Control intervention: Conventional microscopic discectomy}

For microscopic discectomy, the target lumbar level of symptomatic disc herniation is again visualized under C-arm intraoperative fluoroscopy, and a $2.5 \mathrm{~cm}$ midline incision is made over the target level. The paraspinal muscle is detached from the spinous process and the lamina, and detached muscle towing is performed in a minimally invasive fashion through the small skin incision under microscopic visualization. Minimal laminotomy is then performed using a burr and Kerrison punches under microscopic visualization. After the partial removal of the ligamentum flavum under the lamina, discal impingement of the spinal roots and dura is verified. The spinal nerve root is retracted using a root retractor, and the herniated disc is removed by pituitary forceps below the retracted nerve root. Following discectomy, the surgical field is verified for any remnant disc, with the procedure then completed.

\section{Measured outcomes}

A complete description of the time-points at which the data on the primary and secondary outcomes will be collected is provided in Table 1. 
Table 1

Evaluation schedule

\begin{tabular}{|c|c|c|c|c|c|c|}
\hline \multirow{2}{*}{$\begin{array}{l}\text { Visit type } \\
\text { Visit }\end{array}$} & \multirow{2}{*}{$\begin{array}{l}\text { Screening } \\
1\end{array}$} & \multirow{2}{*}{ 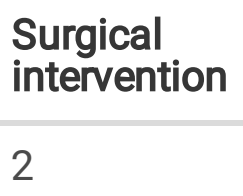 } & \multicolumn{4}{|c|}{ Follow-up } \\
\hline & & & 3 & 4 & 5 & 6 \\
\hline \multirow[t]{2}{*}{ Visit week } & $\begin{array}{l}4-0 \\
\text { weeks }\end{array}$ & $0-2$ days & $\begin{array}{l}2 \\
\text { weeks }\end{array}$ & $\begin{array}{l}12 \\
\text { weeks }\end{array}$ & $\begin{array}{l}24 \\
\text { weeks }\end{array}$ & $\begin{array}{l}52 \\
\text { weeks }\end{array}$ \\
\hline & & & $\begin{array}{l} \pm 5 \\
\text { days }\end{array}$ & $\begin{array}{l} \pm 4 \\
\text { weeks }\end{array}$ & $\begin{array}{l} \pm 8 \\
\text { weeks }\end{array}$ & $\begin{array}{l} \pm 8 \\
\text { weeks }\end{array}$ \\
\hline Informed consent & $\boldsymbol{\square}$ & & & & & \\
\hline Demographics ${ }^{*}$ & घ & & & & & \\
\hline Inclusion/Exclusion & $\boldsymbol{\square}$ & & & & & \\
\hline Randomization & & 口 & & & & \\
\hline Surgery & & $\square$ & & & & \\
\hline MRI (or CT)† & $\mathbf{\square}$ & $\square$ & & & & \\
\hline Simple radiographs & $\boldsymbol{\square}$ & & $\mathbf{\square}$ & $\mathbf{\square}$ & $\mathbf{\square}$ & $\mathbf{\square}$ \\
\hline ODI & $\mathbf{\square}$ & & $\mathbf{\square}$ & $\mathbf{\square}$ & $\mathbf{\square}$ & $\mathbf{\square}$ \\
\hline EQ-5D-5L & $\mathbf{\square}$ & & $\mathbf{\square}$ & $\mathbf{\square}$ & $\mathbf{\square}$ & $\mathbf{\square}$ \\
\hline VAS & $\mathbf{\square}$ & & $\mathbf{\square}$ & $\mathbf{\square}$ & $\mathbf{\square}$ & $\mathbf{\square}$ \\
\hline POSAS & & & & $\mathbf{\square}$ & $\mathbf{\square}$ & $\mathbf{\square}$ \\
\hline Other survey $\ddagger$ & & & $\square$ & $\mathbf{\square}$ & $\mathbf{\square}$ & $\square$ \\
\hline Adverse events & & $\square$ & $\mathbf{a}$ & $\mathbf{\square}$ & $\mathbf{\square}$ & $\mathbf{\square}$ \\
\hline
\end{tabular}

\section{Primary outcome}

The primary outcome will be the efficacy of the surgical intervention (interlaminar full-endoscopic discectomy or conventional microscopic discectomy), measured using the Oswestry Disability Index (ODI) score at 12 months [32, 33]. The ODI is the most useful tool for evaluating patient-reported functional outcome for lumbar spinal disabilities in a clinical setting [32,33]. The ODI evaluates the level of function on activities of daily living for patients with low back pain across the following 10 areas: pain intensity, personal care, lifting, walking, sitting, standing, sleeping, sex life, social life, and traveling. Each section of the ODI is scored on a 5-point scale, with a score of 5 representing the most severe disability. 
The total ODI score was used for analysis, calculated as the sum of the scores across the 10 areas divided by the total possible score, and expressed as a percentage (i.e., multiplied by 100). For all unanswered questions, the total possible score is reduced by five. If the participant checks more than one answer, the highest score is recorded. The ODI will be administered and scored by the assessor and recorded in the eCRF system.

\section{Secondary outcomes}

The following secondary outcomes will be included in the analysis: patient-reported outcomes, clinical outcomes, radiographic outcomes, and adverse events. Patient-reported outcomes are as follows: (1) presence and severity of low back pain and pain radiating to the lower-extremities, measured using a 10point VAS score, ranging from " 0 " (no pain) to "10" (severe pain); (2) quality of life (QOL), measured using the EuroQol-5-dimension-5-level (EQ-5D-5L) questionnaire, which consists of five questions, with the total score ranging between " 0 " and " 1 ," with a higher score indicating a better QOL [34]; (3) satisfaction with the surgery; and (4) walking distance/time and time to return to daily activities after surgery. The following clinical outcomes will be measured: (1) postoperative surgical scarring, measured using the Patient and Observer Scar Assessment Scale (POSAS; version 2.0), which consists of six items scored on a 10-point system, with a score of " 6 " (i.e., a score of ' 1 ' on each item) indicative of normal skin and a score of "60" (i.e., a score of " 10 " on each item) indicative of the "worst scar imaginable" and (2) surgeryrelated variables, namely postoperative drainage $(\mathrm{mL})$, operative time $(\mathrm{min})$, duration of hospitalization (h), and postoperative creatine kinase. The following radiographic outcomes will be obtained: (1) the extent of disc removed and injury to the facet joint, measured using postoperative magnetic resonance (MR) or computed tomography (CT) images and (2) simple radiographs will be used for measuring other complications during the follow-up period. Safety will be evaluated based on the number of adverse events and the severity of these events, as well as surgery-related events. Adverse events will be reported to the surgeon by the participant or assessor and recorded in the electronic database.

Radiographs will be obtained in the anteroposterior, lateral, flexion, and extension views.

Spondylolisthesis and segmental instability at the target surgical level will be scored based on these images. Radiographs will be obtained at baseline and at each of the follow-up time-points. Preoperative spinal MR imaging will be systematically conducted in the sagittal and axial planes to determine the type and location of the disc herniation.

The data on patient-reported outcomes, clinical outcomes, plain radiographs, and adverse events will be collected at baseline and at each follow-up time-point (2 weeks and 3, 6, and 12 months after surgery). Outcomes will be managed and evaluated by the assessor and recorded in the eCRF system (Table 1).

\section{Statistical analysis}

All statistical analyses will be performed using SAS Enterprise Guide 4 (SAS Institute Inc., Cary, North Carolina, USA). The equivalence of baseline variables in both groups will be confirmed statistically prior to the main comparative statistical analyses. 
Modified intention-to-treat (mITT) analysis will be performed, and detailed methods for reducing selection bias due to crossover and loss to follow-up will be used. The mITT strategy will be applied in this RCT, with participants entered in the analysis if they underwent a randomly assigned surgery, thus avoiding the effects of crossover and dropouts, which could break the random assignment to the treatment groups. Participants excluded before or after surgery will not be entered into the analysis or replaced, thus avoiding the risk of bias in allocation concealment. The primary outcome (the ODI score at 12 months post-surgery) will be compared between the two groups. Interlaminar full-endoscopic discectomy will be considered to be equivalent to microscopic discectomy concerning surgical outcomes if the $95 \%$ confidence interval $(\mathrm{Cl})$ of the treatment difference value of the Interlaminar full-endoscopic discectomy group is included in the equivalence limit of 12.8 points

To analyze the time-dependent change in secondary patient-reported and clinical outcomes (i.e., VAS pain scores for the back and lower extremities and ODI, EQ-5D, and POSAS scores), a linear mixed model repeated-measures analysis of variance will be used. Time will be regarded as a categorical variable (2 weeks and 3, 6, and 12 months) and analyzed to evaluate serial changes from baseline, within each group, and between the two groups at each time-point, with a posthoc test used for any significant timeand group-differences identified.

Chi-squared test for categorical variables and Student's t-test for continuous variables will be used for the analyses of other clinical and radiographic outcomes and adverse effects between the two groups. The collected data's distribution will be evaluated using the Shapiro-Wilk test, with a two-sided P-value. Normally distributed continuous variables will be reported as mean and standard deviation (SD), with non-normally distributed continuous variables reported as median and interquartile range. Categorical variables will be reported as count and percentage (\%).

\section{Data management}

Participant data will be anonymized and entered into the iCReaT platform created by the Korean government to allow researchers and investigators to input the research data safely and directly. The iCReaT platform is equipped with a web-based encryption system to protect the research data from unauthorized access and disclosure, and it will be accessible only to the principal investigator and designated statistical analysts. The e-CRF system will be used for this clinical trial. The iCReaT will be managed by specialized clinical research coordinators in each hospital and via a contract with a specialized company with extensive experience in eCRF management. Regarding the monitoring of this clinical trial, both on-site monitoring and in-house monitoring using the electronic data capture system will be conducted by designated monitoring researchers.

\section{Sample size justification}

In this trial, 100 participants will be recruited, with 50 in each group. According to a previous report, the minimal clinically important ODI difference is 12.8 points, with an SD value of 17.1 points at 1-year after endoscopic discectomy $[14,20]$. Based on the equivalence limit of $12.8,50$ participants are required in each group, with an alpha value of 0.05 , a power of 0.90 , a two-sided $95 \% \mathrm{Cl}$, and a loss to follow-up of 
20\%. Power Analysis and Sample Size software (version 15; NCSS, Kaysville, UT, USA) were used to calculate the sample size.

\section{Discussion}

Previous studies have reported that interlaminar full-endoscopic discectomy has similar clinical outcomes and less invasiveness as open microscopic discectomy [18-22]. However, evidence of the advantages of interlaminar full-endoscopic discectomy is limited by the type of research design previously applied, i.e., retrospective, single-center prospective designs [7, 18-25].

This trial will be the most valuable, multicenter, prospective RCT to evaluate and comparatively analyze the efficacy, safety, and applicability of interlaminar full-endoscopic discectomy, compared with open discectomy, in patients with lumbar disc herniation. The quality of the evidence will be improved by an adequate sample size, blinded assessments, and prospective registration from multiple centers to reduce bias; this will ensure that the two approaches are evaluated in an equivalent manner. We anticipate that this high-quality evidence will provide a clear conclusion on the efficacy and safety of interlaminar fullendoscopic discectomy as an alternative option, with the same surgical outcome and less invasiveness, for the treatment of lumbar disc herniation.

\section{Abbreviations}

$\mathrm{Cl}$

confidence interval

CT

computed tomography

eCRF

electronic case report file

EQ-5D-5L

EuroQol 5 Dimension 5 level

iCReaT

internet-based clinical research and trial

mITT

modified intention-to-treat

MR

magnetic resonance

ODI

Oswestry Disability Index

POSAS

Patient and Observer Scar Assessment Scale

QOL

quality of life 
RCT

randomized controlled trial

SD

standard deviation

VAS

visual analog scale

\section{Declarations}

Ethics approval and consent to participate: The design and protocol of this multicenter, assessor-blinded, prospective RCT have been approved by the institutional review board of the five participating hospitals (Catholic University of Korea, Daejeon St Mary's Hospital, DC21ENDOI0021; Catholic University of Korea Seoul St Mary's Hospital, KC21ENDOI0469; Kyung Hee University Medical Center, KHUH 2021; Chungdam Wooridul Spine Hospital, 2021-07-WSH-008; and Wiltse Memorial Hospital, 2021-W03). All participants meeting our eligibility criteria will be required to provide informed consent for the surgery and for the use of their data in research prior to enrollment and randomization.

Consent for publication: Not applicable.

Availability of data and materials: The electronic database server (iCReaT) will not be publicly accessible. Access to the data set is provided only to the Data Management Committee of the Korean Government Research Consortium. The study findings will be published in a peer-reviewed journal.

Competing interests: Jin-Sung Kim is a consultant for RIWOSpine, GmbH, Germany, Stöckli Medical AG, Switzerland and Elliquence, LLC, USA. Jun Ho Lee is a consultant for RIWOSpine, GmbH, Germany. Junseok Bae is a consultant for Joimax, $\mathrm{GmbH}$, Germany. The other authors declare that they have no competing interests.

Funding: This research is supported by a grant from Korea's Health Technology R\&D Project through the Korea Health Industry Development Institute, funded by the Ministry of Health\& Welfare, Republic of Korea (grant number: HC20C0163). The funder had no role in the design of the study or collection, analysis, or interpretation of data or in writing the manuscript.

Authors' contributions:K-JS, L-JH, B-JS, L-DC, P-SM, P-HJ, and L-HJ contributed to the conceptualization, design, writing, and editing of the study protocol. S-SH, K-HJ, C-YS, E-SS, S-SH, H-HJ, K-JY, K-TH, L-W, and $\mathrm{K}-\mathrm{J}$ contributed to the surgical procedure and data collection. All authors approved the final manuscript.

Acknowledgments: We would like to express our very great appreciation to Professor Jin-Sung Kim, Professor Jun Ho Lee, Professor Sang-Min Park, andProfessor Hyun-Jin Park for their valuable and constructive suggestions during planning, obtaining the funding, and development of this research work.

\section{References}


1. Koebbe CJ, Maroon JC, Abla A, El-Kadi H, Bost J. Lumbar microdiscectomy: a historical perspective and current technical considerations. Neurosurg Focus. 2002;13:E3.

2. Muthu S, Ramakrishnan E, Chellamuthu G. Is endoscopic discectomy the next gold standard in the management of lumbar disc disease? Systematic review and superiority analysis. Global Spine J. 2021;11:1104-20.

3. Slepe CJ, Sauer D. Technique of full-endoscopic lumbar discectomy via an interlaminar approach. Eur Spine J. 2018;27:566-7.

4. Sivakanthan S, Hasan S, Hofstetter C. Full-endoscopic lumbar discectomy. Neurosurg Clin N Am. 2020;31:1-7.

5. Wu PH, Kim HS, Jang IT. A narrative review of development of full-endoscopic lumbar spine surgery. Neurospine. 2020;17:20-33.

6. Sharma A, Singh V, Agrawal R, Mangale N, Deepak P, Savla J, et al. Conjoint nerve root an intraoperative challenge in minimally invasive tubular discectomy. Asian Spine J. 2020;15:545-9.

7. Casimiro M. Short-term outcome comparison between full-endoscopic interlaminar approach and open minimally invasive microsurgical technique for treatment of lumbar disc herniation. World Neurosurg. 2017;108:894-900.

8. Wang B, Lu G, Patel AA, Ren P, Cheng I. An evaluation of the learning curve for a complex surgical technique: the full endoscopic interlaminar approach for lumbar disc herniations. Spine J. 2011;11:122-30.

9. Chen KT, Choi KC, Song MS, Jabri H, Lokanath YK, Kim JS. Hybrid interlaminar endoscopic lumbar decompression in disc herniation combined with spinal stenosis. Oper Neurosurg (Hagerstown). 2021;20:E168-74.

10. Son S, Ahn Y, Lee SG, Kim WK. Learning curve of percutaneous endoscopic interlaminar lumbar discectomy versus open lumbar microdiscectomy at the L5-S1 level. PLoS One. 2020;15:e0236296.

11. Li ZZ, Cao Z, Zhao HL, Shang W, Hou S. A pilot study of full-endoscopic annulus fibrosus suture following lumbar discectomy: Technique notes and one-year follow-up. Pain Physician. 2020;23:E497-506.

12. Nakamura JI, Yoshihara K. Initial clinical outcomes of percutaneous full-endoscopic lumbar discectomy using an interlaminar approach at the L4-L5. Pain Physician. 2017;20:E507-12.

13. Choi G, Lee SH, Raiturker PP, Lee S, Chae YS. Percutaneous endoscopic interlaminar discectomy for intracanalicular disc herniations at L5-S1 using a rigid working channel endoscope. Neurosurg. 2006;58:0NS59-68. discussion ONS59-68.

14. Chung AS, Ballatori A, Ortega B, Min E, Formanek B, Liu J, Hsieh P, Hah R, Wang JC, Buser Z. Is less really more? Economic evaluation of minimally invasive surgery. Global Spine J. 2021;11(1_suppl):30S-6S.

15. Liounakos JI, Wang MY. The endoscopic approach to lumbar discectomy, fusion, and enhanced recovery: a review. Global Spine J. 2020;10(2 Suppl):65S-9S. 
16. Xu J, Li Y, Wang B, Lv G, Li L, Dai Y, Jiang B, Zheng Z. Minimum 2-year efficacy of percutaneous endoscopic lumbar discectomy versus microendoscopic discectomy: a meta-analysis. World Neurosurg. 2020;138:19-26.

17. Wang D, Xie W, Cao W, He S, Fan G, Zhang H. A cost-utility analysis of percutaneous endoscopic lumbar discectomy for L5-S1 lumbar disc herniation: transforaminal versus interlaminar. Spine (Phila Pa 1976). 2019;44:563-70.

18. Hamawandi SA, Sulaiman II, Al-Humairi AK. Open fenestration discectomy versus microscopic fenestration discectomy for lumbar disc herniation: a randomized controlled trial. BMC Musculoskelet Disord. 2020;21:384.

19. Ruetten S, Komp M, Merk H, Godolias G. Full-endoscopic interlaminar and transforaminal lumbar discectomy versus conventional microsurgical technique. Spine (Phila Pa 1976). 2008;33:931-9.

20. Song H, Hu W, Liu Z, Hao Y, Zhang X. Percutaneous endoscopic interlaminar discectomy of L5-S1 disc herniation: a comparison between intermittent endoscopy technique and full endoscopy technique. J Ortho Surg Res. 2017;12:162.

21. Choi KC, Shim HK, Kim JS, Cha KH, Lee DC, et al. Cost-effectiveness of microdiscectomy versus endoscopic discectomy for lumbar disc herniation. Spine J. 2019;19:1162-9.

22. Marković M, Živković N, Milan S, Gavrilović A, Stojanović D, Aleksić V, et al. Full-endoscopic interlaminar operations in lumbar compressive lesions surgery: prospective study of 350 patients "ENDOS" Study. J Neurosurg Sci. 2016;30:64:16-24.

23. Li WS, Yan Q, Cong L. Comparison of endoscopic discectomy versus non-endoscopic discectomy for symptomatic lumbar disc herniation: a systematic review and meta-Analysis. Global Spine J. 2021:21925682211020696. doi:10.1177/21925682211020696.

24. Song SK, Son S, Choi SW, Kim HK. Comparison of the outcomes of percutaneous endoscopic interlaminar lumbar discectomy and open lumbar microdiscectomy at the L5-S1 level. Pain Physician. 2021;24:E467-75.

25. Ruan W, Feng F, Liu Z, Xie J, Cai L, Ping A. Comparison of percutaneous endoscopic lumbar discectomy versus open lumbar microdiscectomy for lumbar disc herniation: A meta-analysis. Int $\mathrm{J}$ Surg. 2016;31:86-92.

26. Hofstetter CP, Ahn Y, Choi G, Gibson JNA, Ruetten S, Zhou Y, Li ZZ, Siepe CJ, Wagner R, Lee JH, Sairyo K, Choi KC, Chen CM, Telfeian AE, Zhang X, Banhot A, Lokhande PV, Prada N, Shen J, Cortinas FC, Brooks NP, Van Daele P, Kotheeranurak V, Hasan S, Keorochana G, Assous M, Härtl R, Kim JS. AOSpine consensus paper on nomenclature for working-channel endoscopic spinal procedures. Global Spine J. 2020;10(2 Suppl):111S-21S.

27. Khandge AV, Kim JS. Modified interlaminar endoscopic lumbar discectomy for highly upmigrated disc herniation: A proctorship description of the technique via translaminar route. Neurospine. 2020;17:66-73.

28. Chen KT, Tseng C, Sun LW, Chang KS, Chen CM. Technical considerations of interlaminar approach for lumbar disc herniation. World Neurosurg. 2021;145:612-20. 
29. Lee U, Kim CH, Kuo CC, Choi Y, Park SB, Yang SH, et al. Does preservation of ligamentum flavum in percutaneous endoscopic lumbar interlaminar discectomy improve clinical outcomes? Neurospine. 2019;16:113-9.

30. Chen J, Jing X, Li C, Jiang Y, Cheng S, Ma J. Percutaneous endoscopic lumbar discectomy for L5S1 lumbar disc herniation using a transforaminal approach versus an interlaminar approach: a systematic review and meta-analysis. World Neurosurg. 2018;116:412 - 20.e2.

31. Xu Z, Liu Y, Chen J. Percutaneous endoscopic interlaminar discectomy for L5-S1 adolescent lumbar disc herniation. Turk Neurosurg. 2018;28:923-8.

32. Fairbank JC, Pynsent PB. The Oswestry Disability Index. Spine (Phila Pa 1976). 2000;25:2940-52.

33. Copay AG, Glassman SD, Subach BR, Berven S, Schuler TC, Carreon LY. Minimum clinically important difference in lumbar spine surgery patients: a choice of methods using the Oswestry Disability Index, Medical Outcomes Study Questionnaire Short Form 36, and pain scales. Spine J. 2008;8:968-74.

34. Herdman M, Gudex C, Lloyd A, Janssen M, Kind P, Parkin D, et al. Development and preliminary testing of the new five-level version of the EQ-5D questionnaire (EQ-5D-5L). Qual Life Res. 2011;20:1727-36.

\section{Figures}




\section{Enrollment}

\section{Assessed for Eligibility $(\mathbf{n}=)$}

Included $(\mathrm{n}=)$

- Aged between 20 and 80 years

- Diagnosis of single level lumbar disc herniation Radiating pain to the lower extremities, with a pain score $>4$ (VAS)

- Ability to follow instructions and to provide consent for research participation

- Willingness to comply with the trial follow-up protocol.
- Excluded $(n=)$

- Not meeting inclusion criteria

- Presence of a spondylolisthesis (Meyerding grade $\geq I I)$

- History of lumbar spinal surgery at the same level

- Presence of a degenerative lumbar scoliosis (Cobb angle $>20^{\circ}$ )

- Other spinal diseases (e.g., ankylosing spondylitis, spine tumor, fracture, or neurologic disorders)

- Psychological disorders (e.g., dementia, intellectual disability, or drug abuse)

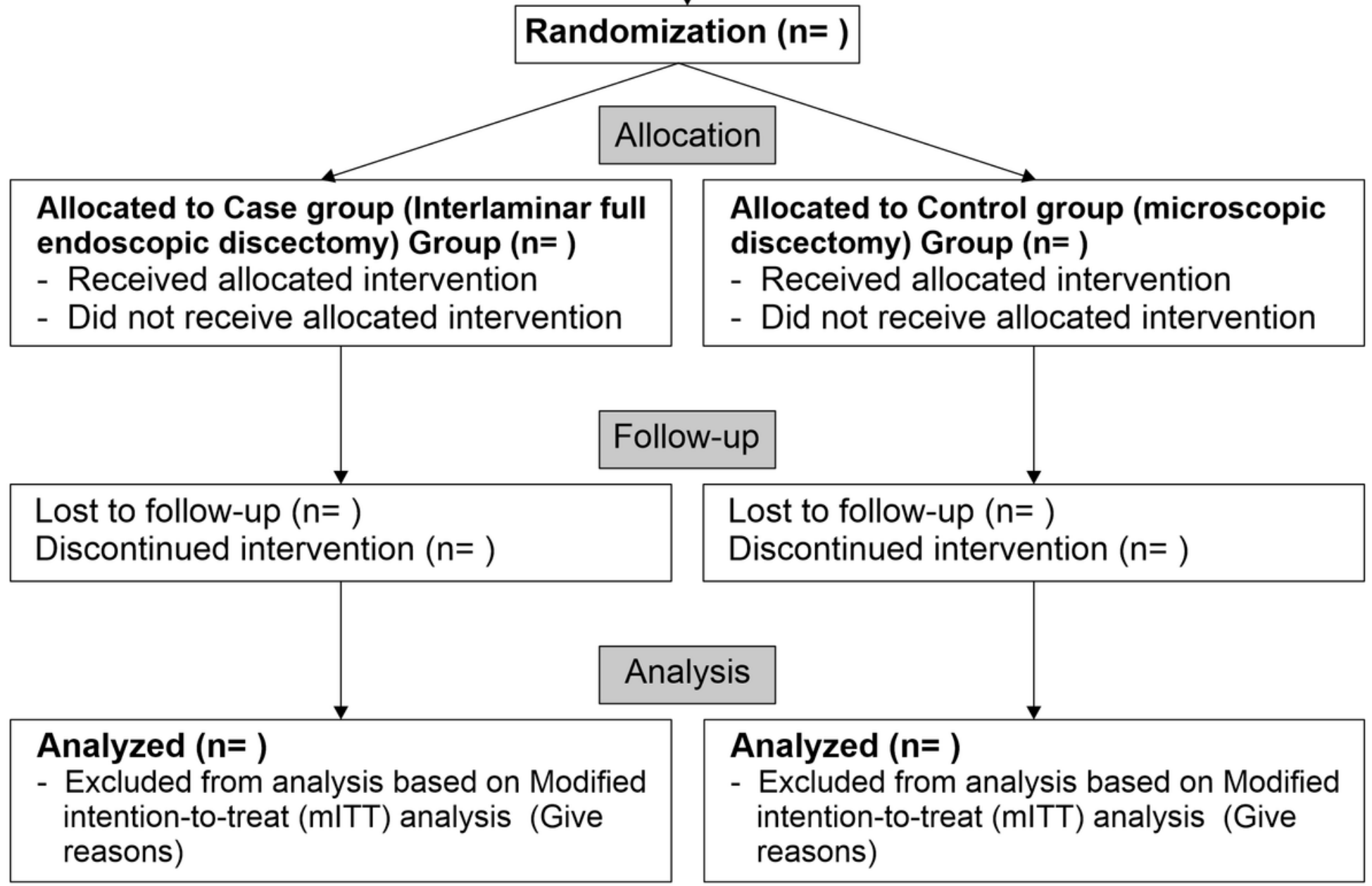

Figure 1

CONSORT study flow diagram for the trial protocol. 
(a)

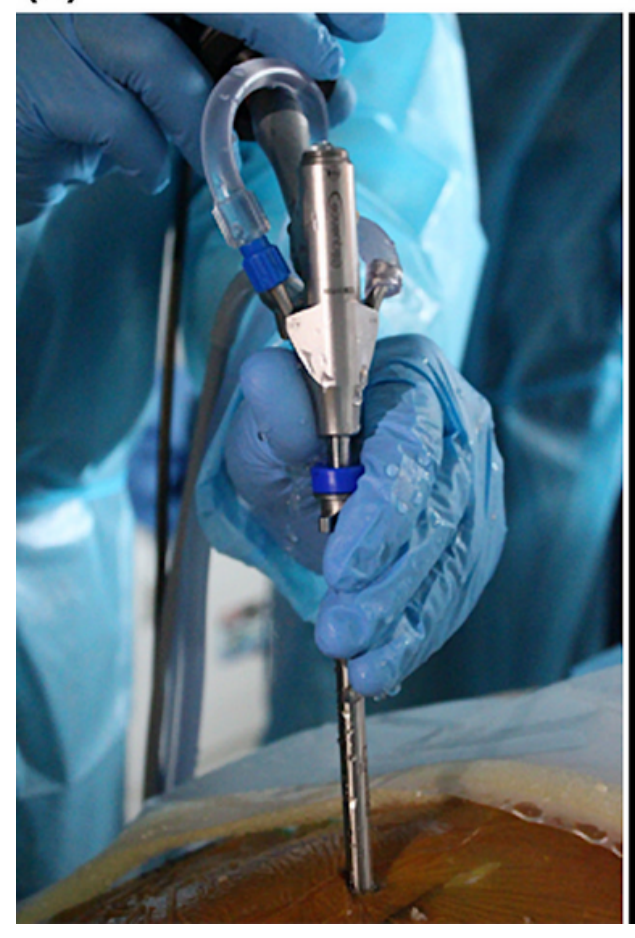

(b)

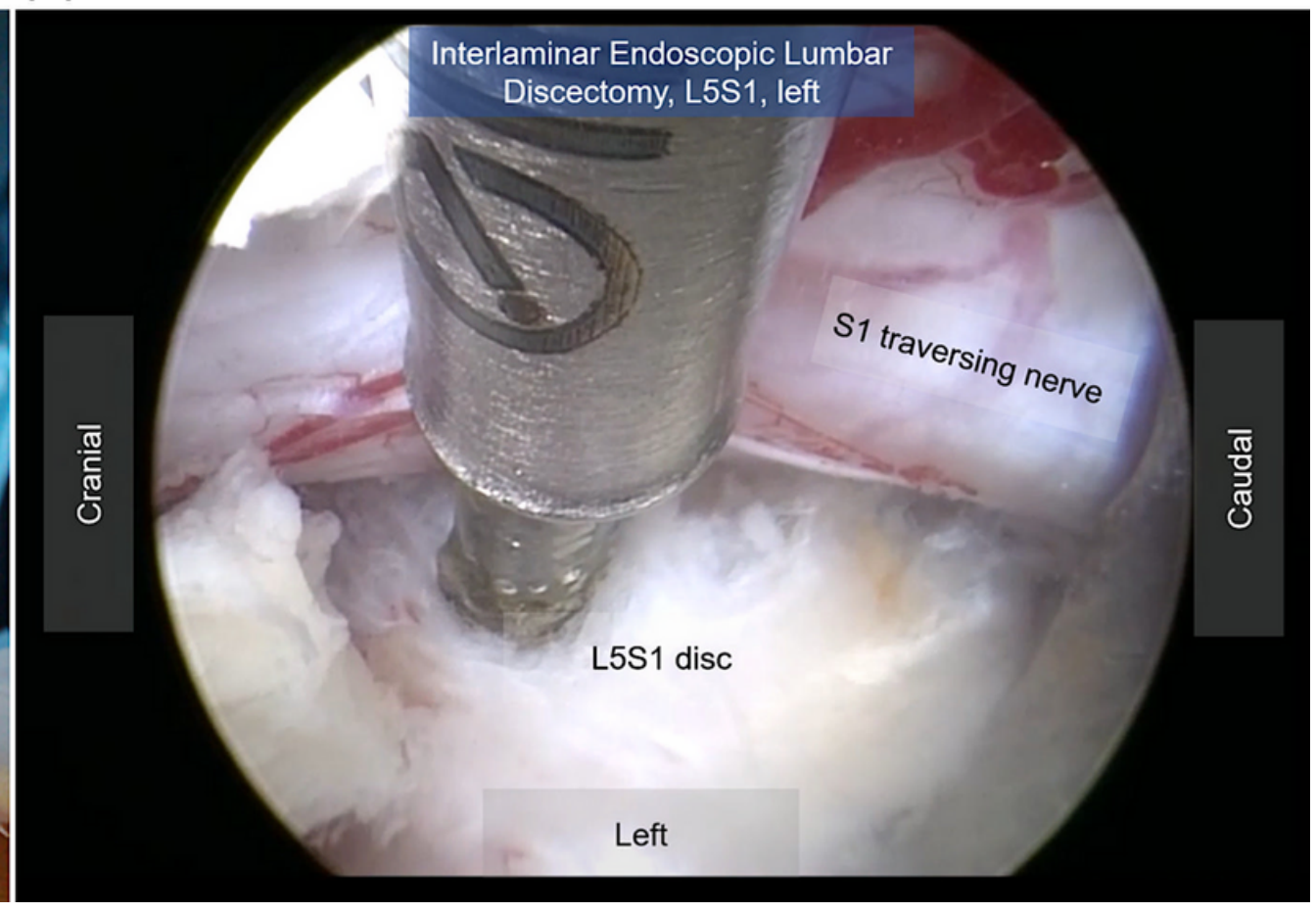

Figure 2

(a) Operative field of interlaminar full-endoscopic discectomy. (b) Intraoperative endoscopic view, showing the disc space and decompressed left S1 nerve root. 